\title{
THE MAGNET FIDUCIALIZATION PROBLEM
}

\author{
Alex Harvey \\ Stanford Linear Accelerator Center \\ Stanford, $C A$
}

Magnets in accelerator beamlines have, for the most part, been made with ferromagnetic poles and traditionally these pole surfaces have been used as the references for external alignment aids, tooling balls, CERN sockets and so on.

This practice assumes that the magnetic field is well-defined by the poles (which fails in the presence of saturation). It also fails in the case of superconducting magnets, which have no tangible poles.

Other difficulties are well-known to those working in the field: the poles of an iron dipole magnet are never perfectly flat, or perfectly parallel. Where, then, is the magnetic mid-plane? The corresponding problem for iron quadrupoles, sextupoles etc, is that there is no unique inscribed circle that is tangent to more than three of these poles. The magnetic axis is then difficult to define. The greater the precision that is sought in the alignment, the more apparent these problems become.

The answer, I believe, is to use magnetic field measurements to establish the references. Most magnets undergo some magnetic measurement before use - they certainly should - and so the opportunity is there to combine fiducialization with magnetic field definition. A substantial part of the Final Focus Test Beam program at SLAC will address this problem.

Meanwhile I would like to use the work of some other SLAC colleagues to illustrate one approach. As part of the installation of the new SLD detector at the Linear Collider, a pair of superconducting triplet quadrupoles will be added as the final focusing elements before the detector. Fiducials (tooling balls) on the outside of the cryostats are referenced to the magnetic axis of the quads in a set-up as shown in Fig. 1 . The probe $P$ is a small rotating coil in a warm-bore insert in the quadrupole. Its center of rotation, $X_{p}, Y_{p}$, can be found by a transit focused on an illuminated target attached to the coil, (see Fig. 2). Analysis of the dipole component of the coil output in a local PC gives the coordinates, $X_{p f}$ and $Y_{p f}$, of the magnetic axis, see Fig. 3 for the schematic. These coordinates are then referenced to sets of tooling balls, BI, B2, B3 and B4, on the outside of the cryostat.

Since the coil has an angular shaft encoder, the azimuthal orientation of the quadrupole field can also be established, and the plot in Fig. 4 shows that this 
magnet lies within \pm 2 mrad except at the ends.

Other magnetic measurement techniques and alignment methods can clearly be used, but the relationship between surveyors and the field measurers must grow closer.

To close, I must acknowledge that the work I have reported is that of Jim Ferrie, Joe Cobb, Dave Jensen, Bill Burgess and others at SLAC. I am grateful for their friendly co-operation. 


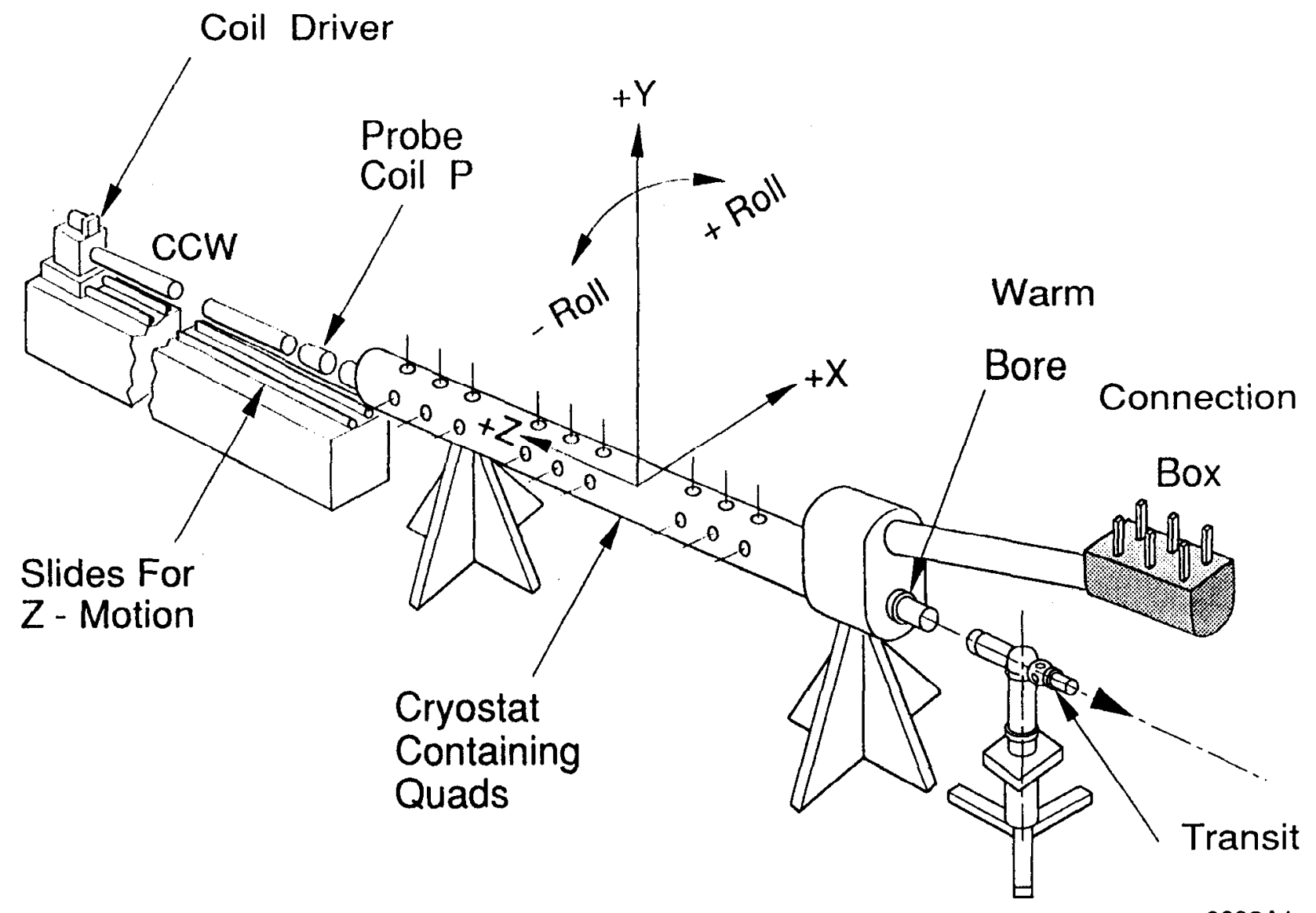

4-90

Figure 1. 


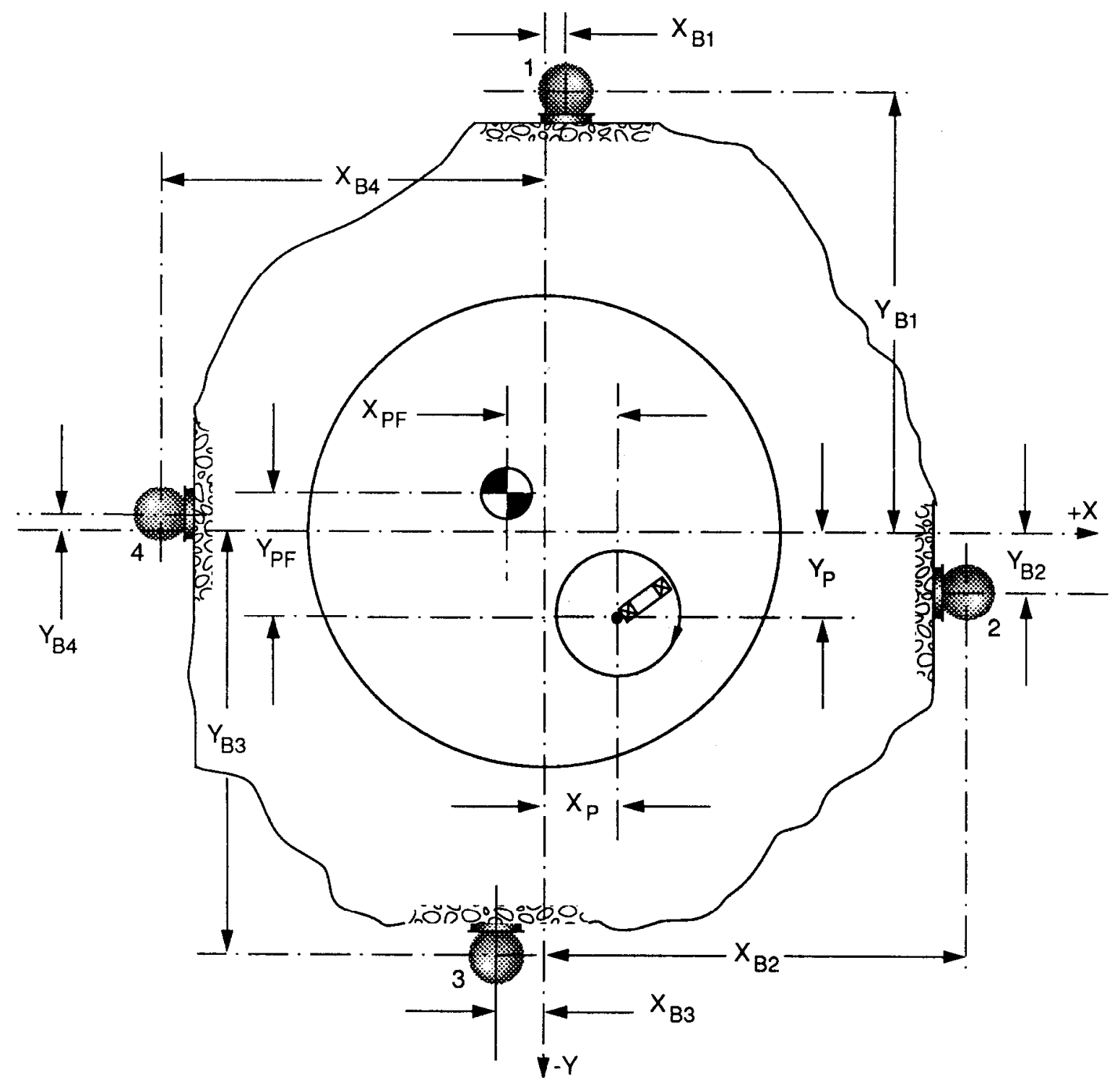

Figure 2. 

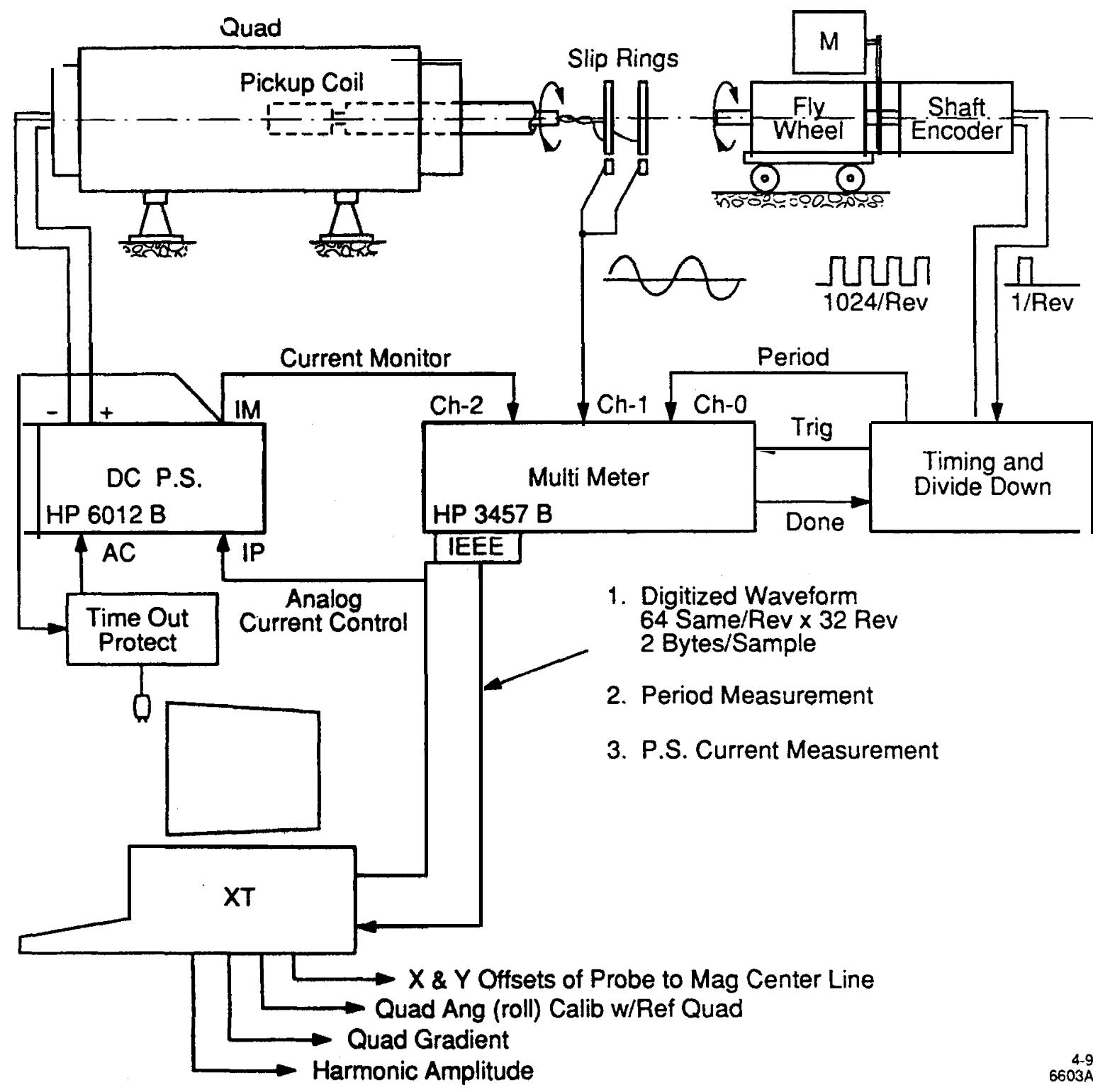

Figure 3. 


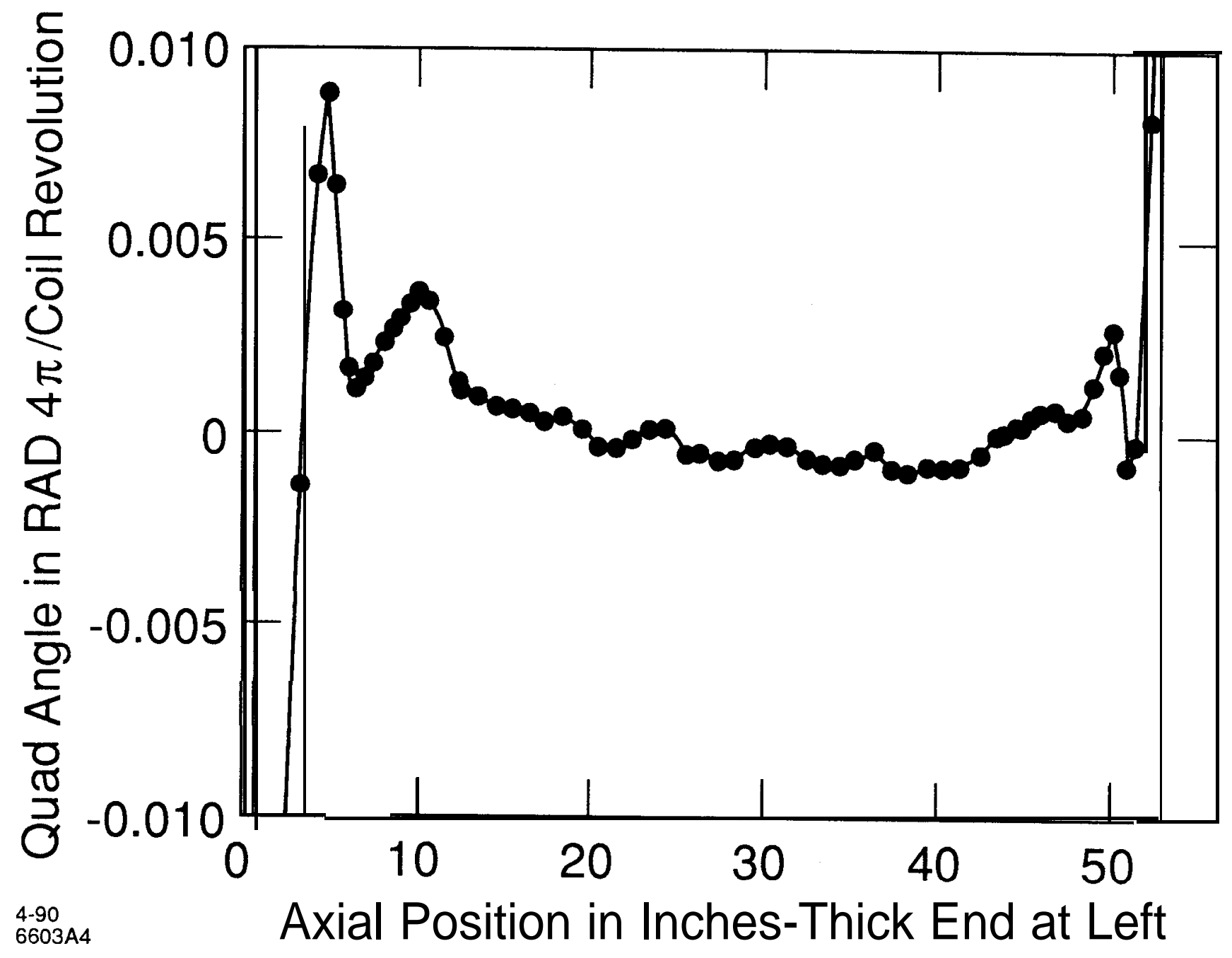

Figure 4. 